\title{
Expression Dominance and Homeolog Expression Bias Upon Chilling Stress in A Hybrid Weed Between the Invasive Sphagneticola Trilobata and the Native S. Calendulacea in South China and its Implications for Invasiveness
}

Wei Wu

Zhongkai University of Agriculture and Engineering https://orcid.org/0000-0003-0542-5043

Guangyan Ni

South China Botanical Garden

\section{Longyuan Wang}

Zhongkai University of Agriculture and Engineering

\section{Hui Zhang}

Zhongkai University of Agriculture and Engineering

\section{yongquan $\mathrm{Li}$}

Zhongkai University of Agriculture and Engineering

\section{Ping Chen}

Zhongkai University of Agriculture and Engineering

Wei Guo ( $\sim$ gwei717@163.com )

Zhongkai University of Agriculture and Engineering

\section{Research article}

Keywords: hybridization, homeolog expression bias, expression level dominance, biological invasion, transcriptome shock

Posted Date: September 15th, 2020

DOI: https://doi.org/10.21203/rs.3.rs-67627/v1

License: (1) This work is licensed under a Creative Commons Attribution 4.0 International License. Read Full License 


\section{Abstract}

Background The role of hybridization or admixture in the biological invasion has been recognized in many invasive species. S ubgenome dominance was reported to occur instantly following the hybridization of divergent genomes, however, investigations on their consequences on physiological response were still scarce. For invasive species, thermotolerance was critical to their range expansions. In this study, a F1 weed between a chilling sensitive invasive S. trilobata and the tolerant native S. calendulacea were treated with consecutive chilling stress, Using physiological assay and RNAsequencing, differential physiological response and transcriptome profile $s$ were compared with a $n$ emphasis on the patterns of the expression dominance and homeolog expression bias in the F1 hybrids.

Results P hysiological assay including content of Malondialdehyde content, superoxide dismutase activity and et al. demonstrated these F1s resembled the chilling tolerant maternal parent S. calendulacea . For both parents and the $\mathrm{F} 1$, the numbers and categories of the enriched pathways or gene ontology terms showed extensive divergence, and an overall resemblance between F1s and S. calendulacea w as observed. For the homeologs with altered expression, expression level dominance was the most prominent pattern. With increased chilling stress, the increased magnitude of up-regulations and decreased magnitude of down-regulations of the Spc- homeologs in the F1s were jointly contributed to the ir resemblance to chilling tolerant parent S. calendulacea . Irrespective of the total homeolog expressive level, increased magnitude of homeolog expression bias toward the chilling tolerant parent $\mathrm{S}$. calendulacea in the F1s also provided another line of evidence for their physiological resemblance.

Conclusion The two Sphagneticola species showed significant differential response upon chilling stress. expression dominance and persistent S. calendulacea homeolog bias in the F1s were attributed to the overall resemblance to the tolerant native species S. calendulacea. Given their vigorous growth, it cations us that the F1 hybrid have great potential to expand northwards and become an awesome weed in the future.

\section{Full Text}

This preprint is available for download as a PDF.

\section{Figures}



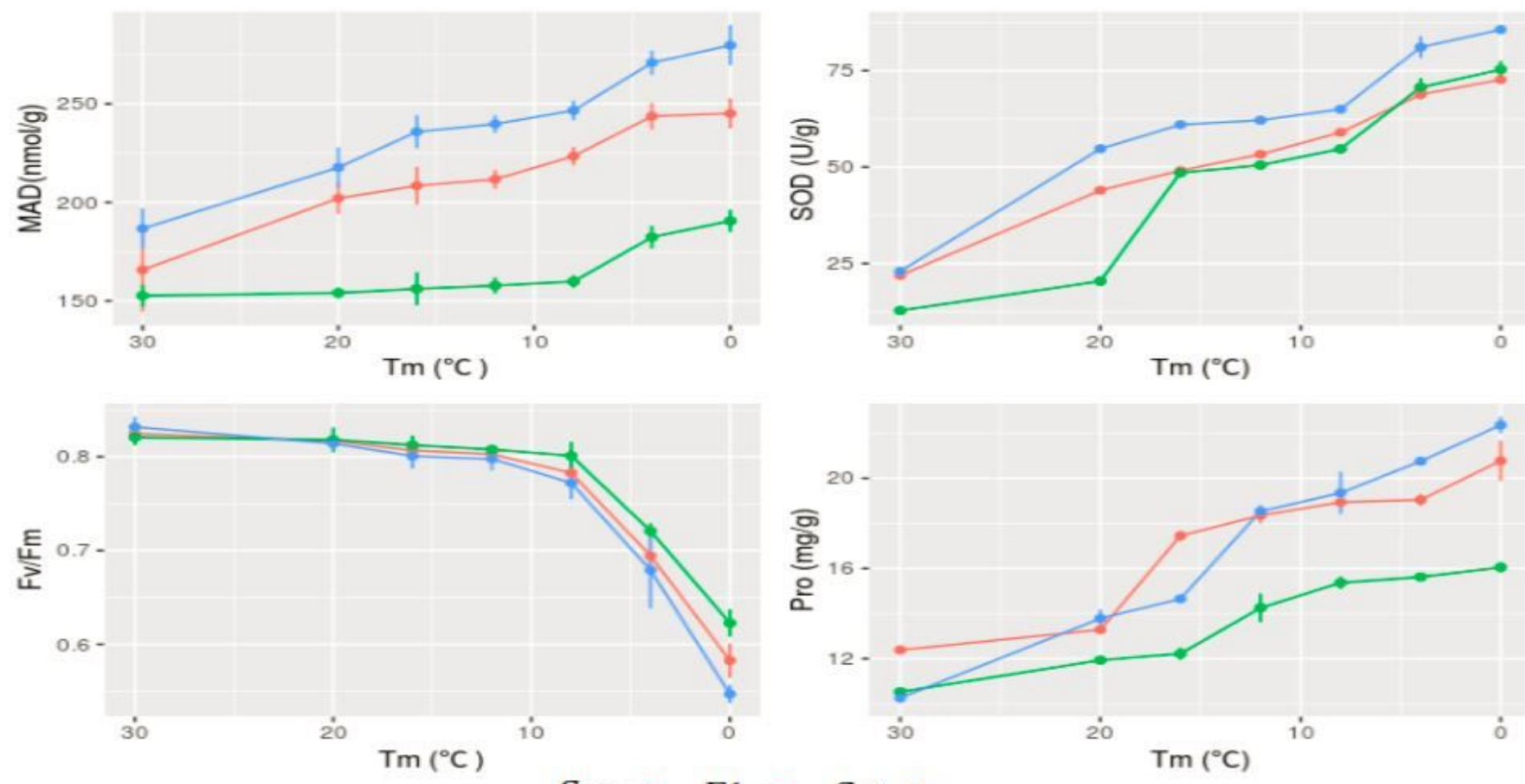

$$
S p c \rightarrow F 1 \multimap S p t \rightarrow
$$

\section{Figure 1}

Physiological responses to consecutive chilling stress among the invasive Sphagneticola trilobata, the native S. calendulacea and their F1s. (a). Malondialdehyde (MDA) concentration; (b) Superoxide dismutase (SOD) activity; (c) Chlorophyll fluorescence, the Maximum quantum yield (Fv/Fm); (d) the concentration of total soluble proteins.
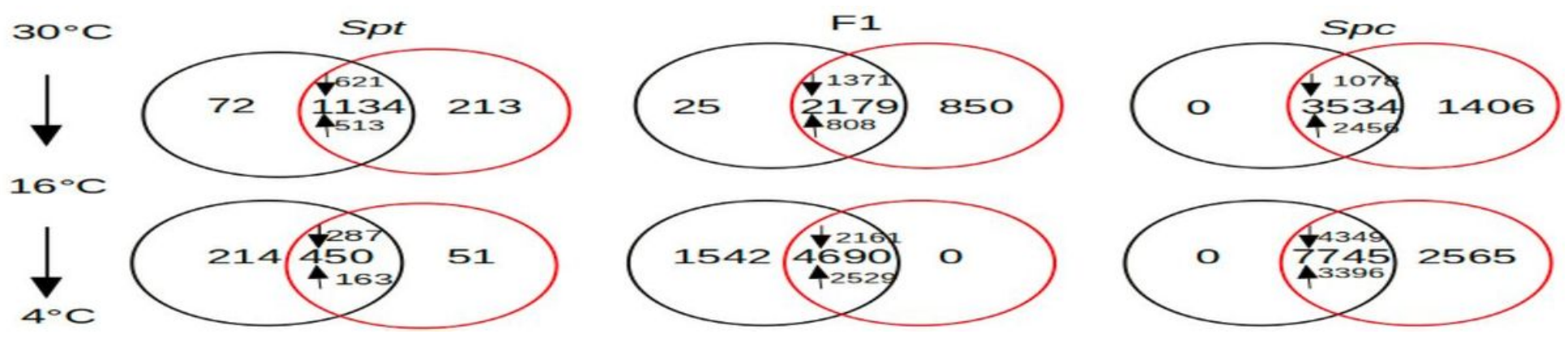

\section{Figure 2}

Venn diagram shown for the shared number of differential expression transcripts between the edgeR (red) and DESeq2 (black) for Sphagneticola trilobata, F1, and S. calendulacea under three gradient temperature chilling from $30^{\circ} \mathrm{C}$ to $4^{\circ} \mathrm{C}$; up-regulated and down-regulated transcripts were marked using upwards arrows and downwards arrows, respectively. Sphagnetila trilobata designated as Spt, S. calendulacea designated as Spc. 


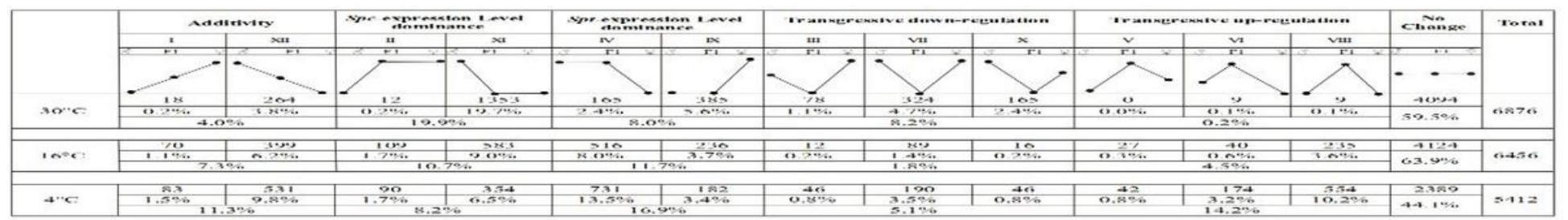

\section{Figure 3}

The thirteen possible expression patterns of $\mathrm{F} 1$ hybrids relative to the paternal parent S. trilobata $(\mathbb{\nabla})$ and maternal parental S. calendulacea ( $\nabla)$ upon consecutive chilling stress $\left(30^{\circ} \mathrm{C}(\mathrm{a}), 16^{\circ} \mathrm{C}(\mathrm{b}) .4^{\circ} \mathrm{C}(\mathrm{c})\right)$. Each pattern is labelled with the same Roman numeral categoried as Rapp et al. (2009). Statistically equivalent expressions were indicated with values on the same horizontal line, whereas statistically significant upand down-regulation were represented by values on higher or lower horizontal lines, respectively. The 13 patterns are binned in five categories representing 'no change', 'Spt-expression level dominance', 'Spcexpression level dominance', 'transgressive expression' and 'additivity', respectively. 

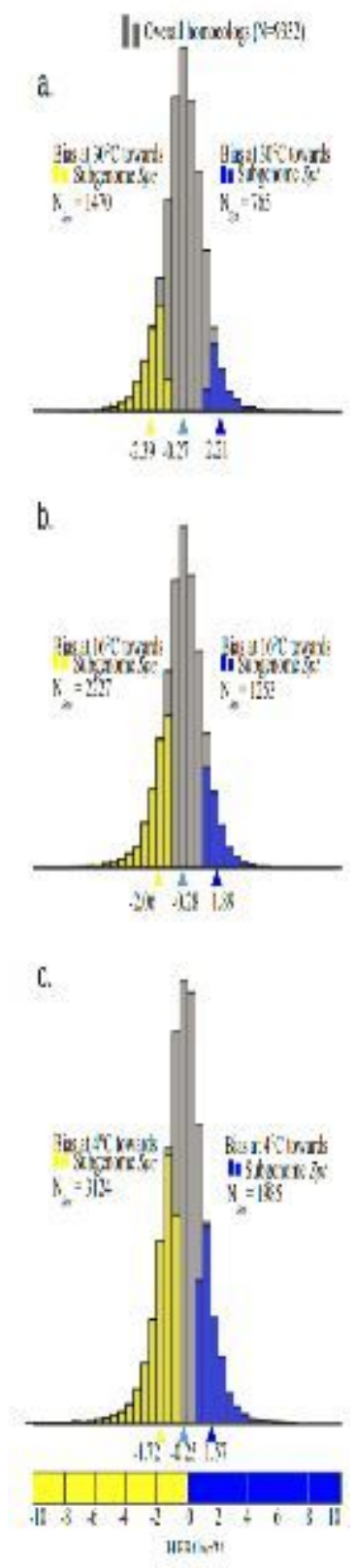

\section{Figure 4}

Likelihood ratio test for homeologs expression bias (HEB) in the F1 hybrids between Sphagneticola trilobata (abbreviated as Spt) and S. calendulacea (abbreviated as Spc) upon consecutive chilling stress $\left(30^{\circ} \mathrm{C}(\mathrm{a}), 16^{\circ} \mathrm{C}(\mathrm{b}) .4^{\circ} \mathrm{C}(\mathrm{c})\right)$. The distribution of expression bias (B) for all testable homeologs in the $\mathrm{F} 1$ indicated by gray histograms, and homeolog pairs with significant expression bias toward Spt-like homeolog were shown in blue histograms, and those with significant expression bias toward Spc-like homeolog were represented by yellow histograms, and the average homeolog bias were marked with corresponding colored triangle values. 

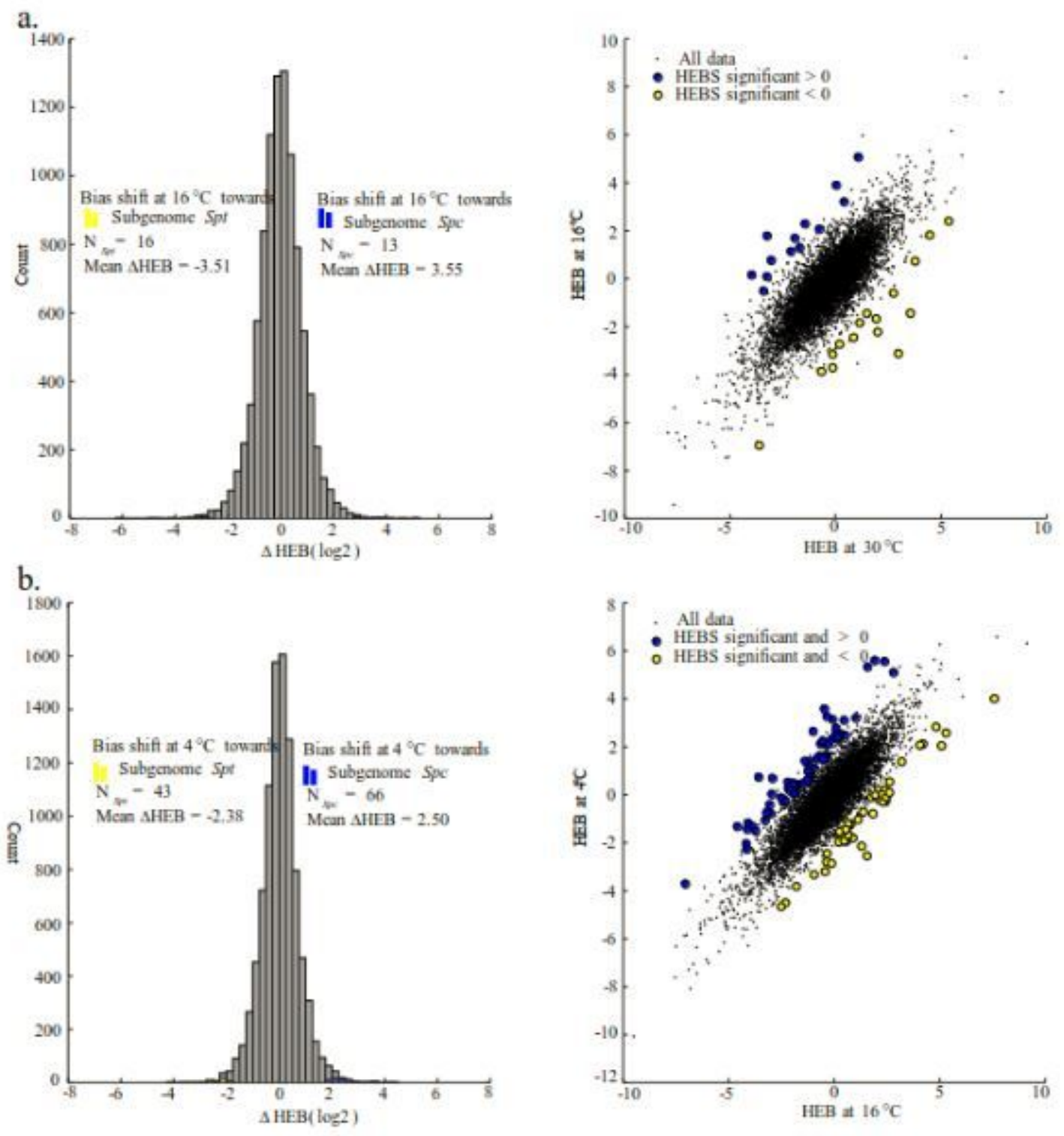

\section{Figure 5}

Likelihood ratio test for changes of homeologs expression bias ( $\triangle \mathrm{HEB}$ ) between consecutive chilling stress ((a) $16^{\circ} \mathrm{C}$ vs $30^{\circ} \mathrm{C}$; (b) $4^{\circ} \mathrm{C}$ vs $16^{\circ} \mathrm{C}$ ) in the $\mathrm{F} 1$ hybrids between Sphagneticola trilobata and $\mathrm{S}$. calendulacea. Histogram plot show the distribution of $\triangle \mathrm{HEB}$, and the counts of homeolog pairs biased toward S. trilobata and S. calendulacea marked using blue and yellow histogram respectively; scatter plot of $\triangle \mathrm{HEB}$ were corresponding to the left histograms. Gene pairs with statistically significant changes in homeolog expression bias marked using the same colors as in the right histograms. Data points in the top-left and bottom-right quadrants represent homeologs where the difference in the bias favors different homeologs at two chilling stress temperatures. On the other hand, the top-right and bottom-left quadrants correspond to homeologs where the difference in bias favors the same homeolog.

\section{Supplementary Files}


This is a list of supplementary files associated with this preprint. Click to download.

- AdditionalFile2.xlsx

- AdditionalFile1.pdf 